\title{
The Potential Perils of Informed Consent
}

\author{
Elizabeth F. Loftus* and James F. Fries
}

Informed consent is supposed to be a good thing, isn't it? Motherhood, apple pie, and informed consent. After all, we don't want the return of the bad old days when unwitting human guinea pigs were experimented upon without knowing what they were getting into. But there is another side to informed consent; in fact, there are perils to informed consent that ought to make the medical establishment think harder before continuing to uncritically accept it as a universal constant.

Some time ago, we wrote an editorial in Science entitled "Informed consent may be hazardous to your health" (1). There we pointed to the rather suggestible nature of the human mind. We know that plying people with certain kinds of information can make them believe that they experienced things that never happened. Studies on the malleability of human memory have shown that people can be led to believe that they saw stop signs instead of yield signs, that they got sick eating particular foods as children, and that they even had experiences that are highly implausible such as witnessing people being demonically possessed or being accosted by the Pluto character on a trip to a Disney resort (2).

Not only can people be led to believe that they experienced events that did not occur, but they can also be led to experience feelings and symptoms that they would not otherwise feel. That's what placebos are all about. When a physician tells a patient or experimental subject that the drug being taken might cause nausea or dizziness, how many people develop the symptoms who wouldn't do so from the drug or the medical procedure alone? We argued that simple inspection of informed consent documents reveals a purpose more directed at

*To whom correspondence should be addressed: Dr. Elizabeth Loftus

University of California, Irvine

2393 Social Ecology 2

Irvine, CA 92697-7085 USA protecting the institution than protecting the experimental subject. We argued that perhaps these individuals would be better off if they got a description of the general level of risk, but detailed risk possibilities, or even very slight risks, ought to be reserved for those who request them. And if the detailed information is given, it ought to be accompanied by a discussion of placebo effects - why they occur, and how to guard against them.

Critics were quick to jump on these ideas, arguing that the "right to full information on matters which may affect our minds and bodies prevails" (3). They accused us of displaying a distressing failure to make a distinction between rights and benefits. We were astonished that our critics would so readily construe a statement intended to be made in favor of human rights as an attack upon such rights. Of course, people have the right to determine what is done to their minds and bodies, but this must extend to symptoms and illness actually caused by poorly executed disclosure. We question, informed by our knowledge of the power of placebos, whether the current legalistic ritual associated with informed consent is the best way to ensure that this human right is protected. We only ask that those whose task it is to formulate informed consent rituals pay some attention to the harm that may be caused by the ritual itself. One harm is the planting of suggested symptoms, some of which can be rather unpleasant, such as anxiety, or downright distressing, such as severe physiological reactions. Would the critics actually endorse the idea that those involved in formulating these rituals have a right to harm people? Patients and subjects might be far less damaged by smaller amounts of balanced information than by mammoth descriptions of remote possibilities. We always advocated supplying the information to those who choose to know all, but to include in the presentation of the "all" a discussion of placebo effects and their potential for adverse reactions. At the time we were immersed in this issue, we 
conducted an unpublished pilot study with patients at Stanford University Medical School who had been diagnosed with scleroderma and were enrolled in an experiment testing the efficacy of a particular drug cocktail (propanolol and alpha-methyldopa). The known side effects of the drugs did include upset stomach, tearfulness, dizziness, and headache. Before beginning the clinical trial involving the drug or placebo, patients received either a standard informed consent message or a "special message." Both messages informed them of possible side effects of the drug, and listed the known side effects, plus some implausible made-up ones that had not been associated with the drug (e.g., ringing in ears, burning sensation in feet).

Some of our subjects received a "special message" as part of their "informed consent." It read as follows: "You should keep in mind one important point about these possible side effects. Research has shown that simply mentioning possible annoying symptoms causes some people to experience these symptoms - even when no drug is taken at all. This happens because mention of the symptoms causes some people to expect that they will experience them, and a person's expectations can then lead to the actual experience. Very few people will actually have these problems and you can help yourself guard against these sorts of discomfort by keeping yourself optimistic and stopping yourself from expecting that side effects are going to happen to you."

Our pilot study revealed, not surprisingly, that subjects experienced side effects, some of which were physiologically unlikely. The suggestion in the informed consent led even those given a placebo to this unpleasant fate. A special message that explained the powerful role that expectations can have in producing unlikely symptoms reduced the reported side effects, and also decreased somewhat the use of medications to treat those unlikely symptoms. Our hope is that future researchers will do a full scale study that tests the impact of variations in informed consent rituals. While our special message may not be the best message to accomplish these hoped-for benefits, and we did not study whether it will work with different kinds of drugs or patients, our preliminary result should pique the interest of future researchers in considering both the positive and negative impacts of information that they ply their subjects and patients with. One size is not likely to fit all. Flexibility in informed consent protocols might convey to patients and subjects their right to as little harm as possible.

\section{REFERENCES}

1. Loftus, EF \& Fries, JF. Informed consent may be hazardous to your health. Science. 1979 April 6;204(4388):11.

2. Loftus EF. Planting misinformation in the human mind: a 30year investigation of the malleability of memory. Learning \& Memory. 2005 Jul-Aug;12(4):361-6.

3. Marcus RB. Kuklick B. Bercovitch S. Uninformed consent. Science. 1979 Aug 17;205(4407):644,646-7.

Elizabeth Loftus is Distinguished Professor at the University of California - Irvine. She holds faculty positions in three departments (Psychology \& Social Behavior; Criminology, Law \& Society; and Cognitive Sciences), and in the School of Law, and is also a Fellow of the Center for the Neurobiology of Learning and Memory. She received her Ph.D. in Psychology from Stanford University. Since then, she has published 22 books (including the award-winning Eyewitness Testimony) and over 450 scientific articles. Loftus's research of the last 30 years has focused on the malleability of human memory. She has been recognized for this research with six honorary doctorates and election to the Royal Society of Edinburgh, the American Philosophical Society, and the National Academy of Sciences. She is past president of the Association for Psychological Science, the Western Psychological Association, and the American Psychology-Law Society.

James F. Fries is Professor of Medicine at Stanford University School of Medicine. He is variously a rheumatologist, gerontologist, pharmaco-epidemiologist, medical ethicist, public policy wonk, clinical epidemiologist, aging mountain climber and author. He has written over 270 scientific articles and 11 books. He is well-known for his original formulation of the Compression of Morbidity paradigm, the introduction of modern patient outcome assessment into medicine, the development of the chronic disease databank model, and longitudinal studies of mortality and morbidity in the fields of arthritis and of aging. 